\title{
Recurrent Pericarditis
}

\author{
JOHN ROBINSON,* M.B., M.R.C.P. ; WALLACE BRIGDEN,* M.D., F.R.C.P.
}

Brit. med. F., 1968, 2, 272-275

The changing prevalence of the aetiological factors in disease is clearly seen in pericarditis. Tuberculosis, pyogenic infection, and rheumatic fever are now relatively rare causes of this condition in Great Britain. Nowadays pericarditis commonly occurs in a relatively benign isolated form, which is often recurrent and of obscure origin (idiopathic pericarditis): virus infection is often implicated but rarely proved. Recurrent pericarditis also occurs as the dominant feature of the postcardiotomy and post-infarction syndromes, and it is common in the so-called collagen diseases. This report concerns observations on a series of patients with idiopathic, postinfarction, and post-cardiotomy pericarditis. It is concluded, as in a previous report on the post-cardiotomy syndrome (Robinson and Brigden, 1963), that there is often good evidence of a hypersensitivity state in these non-pyogenic forms of pericarditis, especially when the disease is recurrent.

\section{The Patients}

This report is based on 20 patients with idiopathic pericarditis, and reference is made to five patients with postinfarction pericarditis and to 16 others with post-cardiotomy syndrome. Patients with pericarditis due to tuberculosis and those with pericarditis due to known connective tissue disease such as rheumatoid arthritis and lupus erythematosus, or to a metabolic disorder such as uraemia, seen during the same period were excluded. All were admitted to hospital at some time during the illness and all had unequivocal evidence of pericardial inflammation. Their ages ranged from 13 to 70 (paediatric cases were not included). In the idiopathic group the average age was 37 and there was a $2: 1$ preponderance of males. In the post-cardiotomy group the average age was 42 and there was a preponderance of females (most operations were on patients with rheumatic mitral valve disease). The average age of the post-infarction group was 55 and all were men. All had had several clinical examinations, electrocardiograms, $x$-ray films, simple haematological tests, and serial E.S.R.s. Most had had tests for antiheart antibodies with tanned red cells (T.R.C.T.), some had had virus studies, and some had had tests for myocardial enzymes in the serum.

\section{Case Reports}

The following short case reports have been selected to illustrate clinical evidence of hypersensitivity and the response to corticosteroid.

\section{Idiopathic Pericarditis}

Recurrent Pericarditis after Vaccination and a History of Allergy. -A married woman aged 48 developed aching retrosternal pain, fever, anorexia, and general malaise four days after vaccination for yellow fever. These symptoms subsided after one week but recurred a week later and resolved again in a few days. Shortly afterwards she was vaccinated for smallpox; and 24 hours later there was a recurrence of fever, severe central chest pain, and breathlessness on slight exertion. The jugular venous pressure (J.V.P.) was con-

* Cardiac Department, the London Hospital, Londen E.1. siderably raised, there was pericardial friction, and the chest $x$-ray film showed moderate enlargement of the heart shadow. E.C.G.s showed ST segment and T-wave abnormalities consistent with pericarditis. Recovery from this attack was spontaneous, but thereafter she had four recurrences over a period of 10 weeks. In addition to her previous symptoms she developed left-sided pleuritic pain and, in the later recurrences, aching in mandibular joints, hips, and knees. There was a past history of recurrent hay-fever and urticaria. She was admitted to hospital at the end of the penultimate attack. The last episode occurred in hospital with the sudden onset of fever and recurrence of central chest pain. Her E.S.R. rose to $68 \mathrm{~mm}$. in one hour and the chest $x$-ray film showed a moderate pericardial effusion. Serum proteins contained increased globulins $(A / G=3.6 / 4.0)$. Virus studies were negative, Tests for antinuclear factor and antiheart antibodies were negative on three occasions. She was given prednisone, starting with $20 \mathrm{mg}$. daily, and became afebrile and free of pain in 24 hours. The E.S.R. fell in a few days and the E.C.G. and $x$-ray appearances also became normal. She continued with $10 \mathrm{mg}$. of prednisone a day for 12 weeks; thereafter she had no further attacks of pericarditis, and has remained well during a follow-up period of three years.

Asthma, Urticaria, and Acute Pericarditis.-A married woman aged 61 had a long history of bronchial asthma. She developed sudden central chest pain and dyspnoea; simultaneously giant urticaria appeared. Pericardial friction and jugular venous hypertension were found on the first day of the illness. Radiography showed a pericardial effusion, and the electrocardiogram had an ST segment elevation. The leucocyte count showed $50 \%$ eosinophils. Prednisone produced a rapid resolution of clinical findings and a normal blood count in two weeks. Though clinical recovery has been complete there were persistent slight abnormalities in the electrocardiogram over a five-year follow-up.

Idiopathic Recurrent Pericarditis, Tamponade, and a History of Asthma.-A youth of 17 had had occasional attacks of asthma since early childhood. Appendicectomy for acute appendicitis, for which he had been given penicillin and streptomycin, was followed after a few days by an asthmatic attack accompanied by fever and aching central chest pain. He recovered after one week and was discharged from hospital. However, four weeks later fever and chest pain with dyspnoea recurred and he was admitted to the London Hospital. His temperature was $100^{\circ} \mathrm{F}$. $\left(37.8^{\circ} \mathrm{C}\right.$.), pulse rate $140 / \mathrm{min}$., and J.V.P. raised to $15 \mathrm{~cm}$. above the sternal angle; there was evidence of tamponade. His E.S.R, was $45 \mathrm{~mm}$. in one hour. $X$-ray examination showed a large pericardial effusion and the E.C.G. widespread ST segment elevation with low voltage in all complexes. Aspiration of 1 litre of pericardial fluid was accompanied by a fall in the venous pressure, a reduction of the tachycardia, and an increase in cardiac output. The fluid was slightly blood-stained ; microscopy showed only red cell debris, and bacteriological and serological studies were negative. The T.R.C.T. for antiheart antibodies was positive. Fever subsided in one week but two weeks later recurred with a rise of the E.S.R. to $43 \mathrm{~mm}$. in one hour. Prednisone starting with $40 \mathrm{mg}$. a day and rapidly reducing to daily maintenance dose of $10 \mathrm{mg}$. a day was given, and he became asymptomatic within 24 hours. His E.C.G. returned to normal and his E.S.R. fell to $8 \mathrm{~mm}$. in one hour, and the T.R.C.T. became negative. He continued prednisone for 20 weeks during which time and after its withdrawal he has remained free from symptoms with no further recurrence of pericarditis.

\section{Post-cardiotomy Syndrome}

A married woman aged 48 complained of pain in the middle of her chest, which became severe on lying down, and aches in various joints two weeks after closed mitral valvotomy. Her temperature 
rose to $101^{\circ} \mathrm{F} .\left(38.3^{\circ} \mathrm{C}\right.$.). Pericardial friction, a raised J.V.P., and slight sacral oedema were found. Her E.S.R. rose to $55 \mathrm{~mm}$. in one hour. Leucocytes were normal and blood cultures were sterile. The T.R.C.T., which had been negative preoperatively, now became positive. The E.C.G., which showed digitalis effect before valvotomy, had greater ST segment depression. Fever subsided and her symptoms disappeared in one week. The T.R.C.T. became negative, and her E.S.R. fell to $12 \mathrm{~mm}$. One week later there was a recurrence of fever, chest pain, and general malaise. The E.S.R. rose to $30 \mathrm{~mm}$. and the T.R.C.T. became positive again. Prednisone (20 mg. a day) was given; she became afebrile and symptom-free in 24 hours. Steroids were discontinued after three weeks. This was followed by a mild fever with pain and swelling of the hands, but these features subsided spontaneously and she has had no further recurrences.

\section{Post-infarction Syndrome}

A man aged 44 developed cardiac infarction while in hospital for investigation of hypertension. Recovery was uneventful for four weeks, but in the fifth week he became febrile and complained of dull central chest pain and pain in the upper abdomen. A persistent pericardial friction rub was heard. His E.S.R. rose to $118 \mathrm{~mm}$. in the first hour and the chest $x$-ray film showed a considerable enlargement of heart shadow. The E.C.G. showed the signs of previous infarction and new ST segment elevation. After two weeks his symptoms subsided, but after a further two weeks they recurred with fever, central chest pain, dyspnoea, and left-sided pleuritic pain. His E.S.R. rose to $53 \mathrm{~mm}$. and $x$-ray examination showed a further increase in the heart shadow. Treatment with prednisone ( $30 \mathrm{mg}$. a day for two days and then progressive reduction) resulted in clinical recovery in 48 hours and the E.S.R, fell to $13 \mathrm{~mm}$. in one hour. After six weeks a further recurrence of pericarditis followed a reduction in the dosage of steroid. Increased dosage (30 mg. of prednisone daily) was rapidly effective and he has remained well since steroid was discontinued, three months after the recurrence.

\section{Clinical Features}

The onset of symptoms could be defined clearly in patients with idiopathic pericarditis, but in those whose illness was a complication of cardiac infarction, cardiac surgery, or some other disease the onset was sometimes ill-defined. In most patients the disease was fully developed in one or two days; in a few the onset was rapid (a few hours), but it was never as abrupt as in pulmonary embolism or in some cases of cardiac infarction. In the post-infarction and post-cardiotomy syndromes the interval before the onset of pericarditis was seldom less than 10 days and in the majority it occurred between the second and fourth weeks. However, in one-fifth of the patients with post-cardiotomy syndrome pericarditis first occurred several weeks after operation; the longest period was 18 months; in one patient it occurred two years after salient cardiac infarction.

Pain in the centre of the chest was the first symptom in the majority, and it was usually described as a sensation of heaviness ; often, however, it became sharp and stabbing on taking a deep breath. Unlike the pain of cardiac infarction it was mostly made worse by lying flat, by stretching the chest, and sometimes by swallowing. None of the 41 patients ever had pain in the arms, but pain across the shoulders was common. It was rarely very severe and usually subsided in a few days. Pleuritic pain on one side or the other occurred at some time in half the patients.

Arthralgia with malaise was usual. Though pain in large joints was rare, several patients complained of aches in small joints. Joint swelling was seen in only one patient. Dyspnoea was not an impressive feature, and was serious in only two patients who had evidence of tamponade.

A past history of hypersensitivity was of interest and possibly significant in several patients. Seven of the 20 patients who had idiopathic pericarditis had had either asthma or urticaria or other skin trouble at some time in the past, and one had a remarkable but transient eosinophilia during the attack of pericarditis (see Case Reports).

Fever varying between 99 and $101^{\circ} \mathrm{F}$. $\left(37.2\right.$ and $38.3^{\circ} \mathrm{C}$.) occurred early and persisted for a few days in all except three patients. Pericardial friction was an early sign, but was often of brief duration; for this reason it was heard most often in the post-cardiotomy and post-infarction patients who were already under observation. The jugular venous pressure was raised slightly in the majority of patients. However, it exceeded $10 \mathrm{~cm}$. in only two patients who had other features of tamponade.

The E.S.R. was greatly raised in every patient during the attack, usually between 50 and $80 \mathrm{~mm}$. in one hour. There was evidence from a few cases that the rise preceded the onset of symptoms. Elevation of the E.S.R. persisted throughout the febrile and symptomatic period but fell rapidly thereafter, and thus appeared to be a sensitive, and possibly the best, indicator of the activity of the disease whatever its cause.

The E.C.G. was abnormal at some time during the illness in all the patients. The well-known features of ST segment elevation in several leads with lowering of $T$ waves was common, but transient $\mathrm{T}$-wave inversion sometimes occurred without ST abnormalities. The voltage of the QRS complex was conspicuously reduced only when there was clear evidence of a pericardial effusion. Disturbances of conduction were not seen, and only one patient, who possibly had myocarditis as well as pericarditis, had an arrhythmia. E.C.G. abnormalities due to pericarditis after cardiotomy or cardiac infarction were most difficult to analyse, and only careful perusal of serial tracings showed an adverse change. Thus in cases of delayed pericarditis after a transventricular mitral valvotomy $T$-wave inversion in anterior chest leads became more pronounced. Radiology contributed little to diagnosis; slight enlargement of the heart shadow was common; great enlargement was rare and occurred only in patients who had clear clinical evidence of a large effusion. Many patients had a small pleural effusion; none had evidence of disease in the lung parenchyma.

\section{Immunological Investigations}

Tests for antiheart antibodies using the tanned sheep red cell technique were carried out in 33 patients as previously described (Robinson and Brigden, 1963) (see Table). A reproducible titre of $1: 80$ or higher was accepted as positive in our method.

Tanned Red Cell Haemagglutination Test for "Antiheart" Antibodies in

\begin{tabular}{|c|c|c|c|c|}
\hline & \multirow{2}{*}{$\begin{array}{c}\text { No. } \\
\text { of } \\
\text { Patients }\end{array}$} & \multirow{2}{*}{$\begin{array}{c}\text { No. } \\
\text { Tested }\end{array}$} & \multicolumn{2}{|c|}{ T.R.C.T. Result } \\
\hline & & & Pos. & Neg. \\
\hline $\begin{array}{l}\text { Idiopathic pericarditis } \\
\text { Post-infarction syndrome } \\
\text { Post-cardiotomy syndrome }\end{array}$ & $\begin{array}{r}20 \\
5 \\
16\end{array}$ & $\begin{array}{r}15 \\
4 \\
14\end{array}$ & $\begin{array}{r}6 \\
3 \\
12\end{array}$ & $\begin{array}{l}9 \\
1 \\
2\end{array}$ \\
\hline
\end{tabular}

In the idiopathic group positive results were obtained from six of the 15 cases tested. There were no differences in the clinical features, past history, or number of recurrences in patients with positive tests and those with negative results.

In those with the post-infarction syndrome positive results were obtained in three or four who were tested. Titres were of the same order as in patients with the post-cardiotomy syndrome and the return to negativity with resolution of the attack was also similar.

Positive results were obtained in 12 of the 14 patients tested who developed the post-cardiotomy syndrome. In all except one of these the test became negative with clinical resolution; however, in some cases a late return to positivity occurred and heralded the onset of clinical recurrence. In one patient positive tests were obtained for several months though clinical evidence of active disease did not appear. 


\section{Clinical Course}

Recurrence of pericarditis after resolution of the first attack was common in all types. In the idiopathic group 17 out of 20 patients had recurrences, most of which occurred in the six months after the initial attack, but some continued to recur thereafter and in one patient there were episodes for three years. Patients with the post-infarction syndrome showed the same tendency to recurrent pericarditis (four out of five), but in the post-cardiotomy syndrome the number of patients who had recurrences was slightly less (12 out of 16), four having had only one attack over a prolonged period of observation. All are well with the exception of one who died suddenly five years after the attack of pericarditis (cause unknown).

Corticosteroid was given to 14 patients in the whole seriesalways after more than one recurrence of pericarditis. Most were given $10 \mathrm{mg}$. of prednisone three times a day for two days (a few were given larger doses). Thereafter the dose was rapidly reduced to 10 to $15 \mathrm{mg}$. a day and stopped after 12 weeks. In some the effective level of steroid dosage could be ascertained within a few milligrams. Thus in one patient who had several recurrences of pericarditis after cardiac infarcation $12.5 \mathrm{mg}$. was consistently effective, but reduction to $10 \mathrm{mg}$. always resulted in recurrence.

\section{Discussion}

The clinical features of pericarditis are too well known to merit comprehensive comment; however, in patients who have prolonged pain the diagnosis from cardiac infarction, the importance of which requires no emphasis, continues to be a problem. Though no single clinical feature excludes cardiac infarction, the influence of chest movement, posture, and swallowing on the pain and its absence in the arms in pericarditis is stressed. Indeed, we have seen no patient with pain in the arms whose disease was confined to the pericardium and whose subsequent history showed that the condition was not due to coronary disease. A further important pointer to isolated pericarditis is the wide distribution of minor abnormalities in all leads of the E.C.G. associated with an E.S.R. which is raised above the levels usually found in cardiac infarction, and in the absence of elevated muscle enzymes in the serum. Theoretically the greatest difficulty must arise when a small and possibly superficial infarct causes severe pericarditis ; we have, however, not knowingly encountered this condition.

The differentiation of the so-called idiopathic form from tuberculous pericarditis is most important. Error has serious consequences, for the greater the delay in treatment of the tuberculous form the higher the mortality and the greater the risk of subsequent constriction, while prolonged antituberculous treatment given unnecessarily is clearly undesirable. Clinical features such as the insidious onset and the prolonged and often hectic fever of tuberculosis are in contrast to the subacute onset and often rapid resolution followed by recurrence of the idiopathic form. When doubt exists examination of the pericardial fluid is crucial (Schrire, 1967). Pericardial paracentesis was performed in our patients only when there was a serious possibility of tuberculosis, acute bacterial infection, or a malignant cause and when signs of tamponade appeared.

None of the patients in this series developed constriction, although this has been reported in idiopathic pericarditis (Krook, 1954). Apart from constriction, pericardectomy has been advocated for the prevention of recurrent attacks of pericarditis (Jamplis, 1964). This in our experience is unnecessary, and, furthermore, Goldfarb et al. (1966) reported a case of recurrent fever and "pericardial" pain after pericardectomy, supporting the view that the pericarditis is one manifestation of a hypersensitivity state rather than the primary cause. No patient in this series required pericardectomy: it is thus possible that judicious and early use of steroids makes this procedure unnecessary. None of our patients has died from pericarditis, though fatalities have been recorded (Swan, 1960 ; Liu and Garcia, 1965). Tamponade is the great danger, and its recognition demands aspiration of fluid followed by corticosteroid if the diagnosis of idiopathic, post-cardiotomy, or postinfarction pericarditis is correct. We believe that fatalities said to be due to idiopathic pericarditis associated with serious arrhythmia or heart failure other than tamponade are the result of myocarditis.

Pericarditis may be due to many processes known to cause disease in man, including many forms of infection, autoimmune disease causing connective tissue disease, metabolic disorders, trauma, and neoplasia. However, it seems that while some of these processes may initiate the pericarditis the subsequent clinical reaction tends to have a common pattern which is independent of the cause. The events preceding the development of "idiopathic" pericarditis are clearly of great importance in solving the problem of the causation. Early reports stressed the frequency of preceding respiratory infection (Gilley et al., 1951), but we have not been able to confirm this relation. However, a proportion of subjects exposed to epidemics of Coxsackie infection have developed pericarditis (Hedlund et al., 1962), and other virus infections have been shown to cause pericarditis. Penicillin sensitivity (Schoenwetter and Silber, 1965), serum sickness (Goldman and Lau, 1954), and tetanus antitoxin (Fox and Messeloff, 1942) have caused pericarditis which, in one of our patients, was clearly related to yellow fever and smallpox vaccinations (see above). Another of our patients had recently had appendicectomy, as had one in the series reported by Feder et al. (1950), but antibiotic therapy was used in these cases and may have been the precipitating factor. We have found that a history of allergy is not uncommon, suggesting that pericarditis may be concerned with hypersensitivity.

The view that the reaction pattern in recurrent pericarditis is associated with an abnormal state of hypersensitivity in the immunological sense is supported by the following features:

(1) There is always delay before the development of the reaction (when the initiating stimulus can be recognized and dated).

(2) Some patients have a past history suggesting a predisposition to allergy; thus some have had asthma, hay-fever, or urticaria. Furthermore, the post-cardiotomy syndrome is apparently much more common after operations for rheumatic heart disease than after operations for congenital defects, suggesting that those who have shown an abnormal immunological response in the past are more likely to respond abnormally to cardiotomy.

(3) Some patients have evidence of an associated serositis, such as pleural pain or arthralgia.

(4) Analogy with certain connective tissue diseases known to be associated with an inappropriate immunological reaction is close in some respects. Thus all patients with systemic lupus erythematosus have pericarditis at some time (Brigden et al., 1960 ), and some $40 \%$ of patients with rheumatoid arthritis have pericarditis (Sokoloff, 1964) ; furthermore, in both conditions there is a cyclical tendency and recurrent bouts of pericarditis are common.

(5) All patients appear to have a high E.S.R. during the attack-higher than that associated with, for example, a brief viral infection, but often of the order found in the known hypersensitivity diseases during phases of activity.

(6) There is a high frequency of positive immunological tests during active phases of the disease (see Table).

(7) Corticosteroid produces a rapid therapeutic response which appears to be suppressive as in connective tissue diseases. Furthermore, dosage may be critically adjusted to achieve complete suppression.

\section{Summary}

The clinical features of benign recurrent pericarditis based on a study of 20 patients are described and reference is made to observations on a further 21 patients with pericarditis asso- 
ciated with the post-infarction syndrome and post-cardiotomy syndrome. The problems of differential diagnosis are discussed. Cardiac tamponade was the most serious complication ; but this was uncommon and was rapidly relieved by aspiration of pericardial fluid. No patients required pericardectomy, possibly because corticosteroids brought rapid resolution or possibly because constriction is so rare that it was not encountered in any one of these 41 patients. Furthermore, corticosteroid therapy effectively prevents recurrences, which are common in all forms of "benign" pericarditis.

"Antiheart" antibodies were detected in almost all of the post-infarction and post-cardiotomy cases and in one-third of the idiopathic group. It is concluded that recurrent pericarditis may be provoked by a variety of noxious agents, which lead, in susceptible individuals, to an abnormal immune reaction independent of the primary cause.
REFERENCES

Brigden, W., Bywaters, E. G. L., Lessof, M. H., and Ross, I. P. (1960), Brit. Heart Y., 22, 1 .

Feder, I. A., Hoffiman, J., and Sugar, H. (1950). Amer. F. med. Sci., 220 144.

Fox, T. T., and Messeloff, C. R. (1942). N.Y. St. F. Med., 42, 152.

Gilley, E. W., McCord, M. C., and Taguchi, J. T. (1951). Amer. ? med. Sci., 222, 249 .

Goldfarb, B., Gold, D., Latts, E., Wexler, H., and Wang, Y. (1966) Circulation, 33, 283

Goldman, M. J., and Lau, F. Y. K. (1954). New Engl. 7. Med., 250, 27 S Hedlund, P., Lycke, E., and Tibblin, G. (1962). Acta med. scand., 172 323.

Krook, H. (1954). Acta med. scand., 148, 201.

Jamplis, R. W. (1954). Amer. Y. Surg., 108, 191

Jamplis, R. W. (1954). Amer. F. Surg., 108, 191.
Liu, H. Y., and Garcia, R. (1965). Amer. Heart 7., 69, 677.
Robinson, J., and Brigden, W. (1963). Brit. med. F., 2, 706.

Schoenwetter, A. H., and Silber, E. N. (1965). 尹. Amer. med. Ass., 191 672 .

Schrire, V. (1967). Aust. Ann. Med., 16, 41.

Sokoloff, L. (1964). Mod. Conc. cardiovasc. Dis., 33, 847.

Swan, W. G. A. (1960). Brit. Heart F., 22, 651.

\title{
Effects of Cortisol Deficiency on the Electrocardiogram
}

\author{
M. HARTOG, ${ }^{*}$ D.M., M.R.C.P. ; G. F. JOPLIN,* PH.D., M.R.C.P.
}

Brit. med. F., 1968, 2, 275-277

Abnormalities of the clectrocardiogram (E.C.G.) have long been known to occur in Addison's disease (Coelho, 1932 ; Delius and Opitz, 1935), and an extensive analysis of such abnormalities has been reported by Somerville et al. (1951). Changes in the E.C.G. in hypopituitarism have also been described (Sheehan and Summers, 1949); these abnormalities were reversed by treatment with thyroid hormone and must therefore have been caused by thyroid deficiency. However, others have noted that in some hypopituitary patients the E.C.G. reverted to normal if only cortisone was given (Kosowicz and Roguska, 1963).

We have had the opportunity of studying the effects of cortisol deficiency on the E.C.G. in euthyroid patients with cancer in whom an attempt had been made to ablate the pituitary by needle implantation of radioactive yttrium (Fraser and Joplin, 1961). Since the highest degree of destruction of the gland affords the best chance of a remission of the disease (Joplin, 1965), it was essential to assess pituitary function after operation so that a second implant could be performed in the event of an incomplete ablation. As part of this assessment corticosteroid therapy was withdrawn after operation, and the effect of this withdrawal on the patients' general condition and on serial water diuresis tests was observed. The majority of these withdrawals were done within three weeks of operation, which was too early for significant hypothyroidism to have developed. In those subjects in whom a withdrawal at a later date was performed an assessment of their thyroid state had previously been made, and those thought to be hypothyroid had been treated with thyroxine. Thus the effect of cortisol deficiency alone on the E.C.G. could be followed, both during corticosteroid withdrawal and during subsequent repletion, where this was necessary.

\section{Patients Studied and Methods Used}

Patients Studied.-The patients consisted of 26 women suffering from disseminated breast cancer and two men with disseminated cancer of the prostate.

Clinical Management.-In most cases cortisone administration was discontinued on the 10th postoperative day. In some a withdrawal was performed during a later admission for general

* Royal Postgraduate Medical School, London W.12. assessment. After withdrawal of cortisone a careful watch was maintained for clinical evidence of corticosteroid deficiency such as anorexia, nausea, lethargy, fever, or hypotension. As soon as corticosteroid deficiency was diagnosed the patients were rapidly repleted with prednisone. The standard repletion regimen was $20 \mathrm{mg}$. of prednisone by mouth initially, followed by 5-mg. hourly for six doses, and then $5 \mathrm{mg}$. four-hourly until the patient was fully recovered, which usually took less than 24 hours. Adrenocortical function was assessed by means of serial water diuresis tests in which a litre of water was administered. Normal subjects excrete over $75 \%$ in four hours, whereas patients with adrenal insufficiency usually excrete less than $40 \%$ during this period (Joplin, 1965). In some patients estimations of urinary 17-oxygenic steroids were performed by the method of Norymberski et al. (1953). However, many of these seriously ill cancer patients had low levels of 17-oxygenic steroids before operation, so that the usefulness of these estimations during the period of corticosteroid withdrawal was limited.

E.C.G. Recording.-Complete E.C.G.s were taken on all patients before operation; only those whose tracings were normal before operation have been included in this paper. Tracings were recorded serially throughout the period of cortisone deprivation, and also during the subsequent phase of repletion when this was performed. The majority of the E.C.G.'s were taken with a Mingograf (Elmquist) machine. This instrument has a high input impedance, which minimizes differences in resistance between skin and electrodes. Owing to the difficulty of obtaining reproducible tracings of the precordial leads, our analysis is based solely on the standard and unipolar limb leads. Most of the E.C.G.s have been analysed and measured by a cardiologist who was unaware at the time of the clinical state of the patient. The components of the E.C.G. which were measured were the $P-R$ interval, the width and amplitude of the QRS complex, the Q-T interval, and the amplitude of the $T$ waves. The height of the $T$ wave in the serial tracings was expressed as a percentage of the height before cortisone withdrawal, this value being taken as $100 \%$. In each case the E.C.G. was reviewed as a whole to ensure that apparent changes in the size of the $T$ wave were not due to obvious changes in the vector. In all patients studied any change of the $T$ wave in the axial limb lead was reflected throughout the other available leads. 